
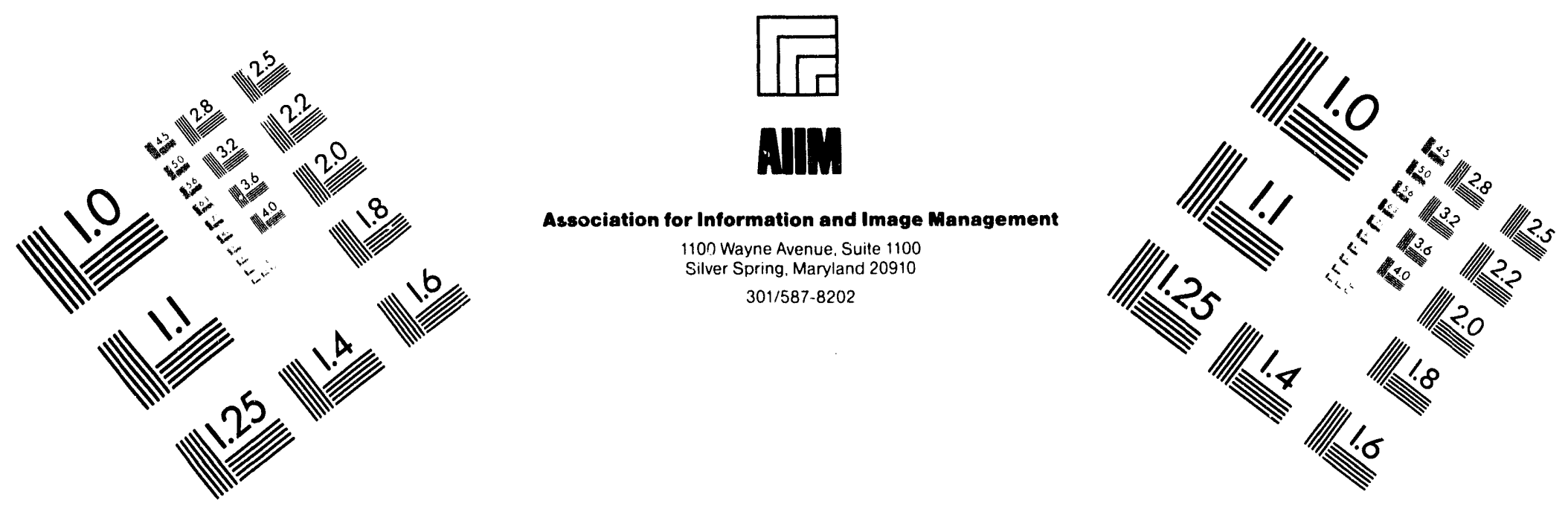

\title{
Centimeter
}

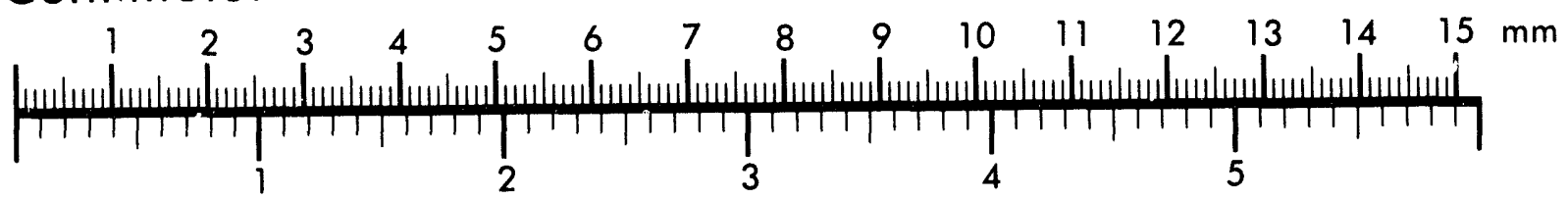

Inches
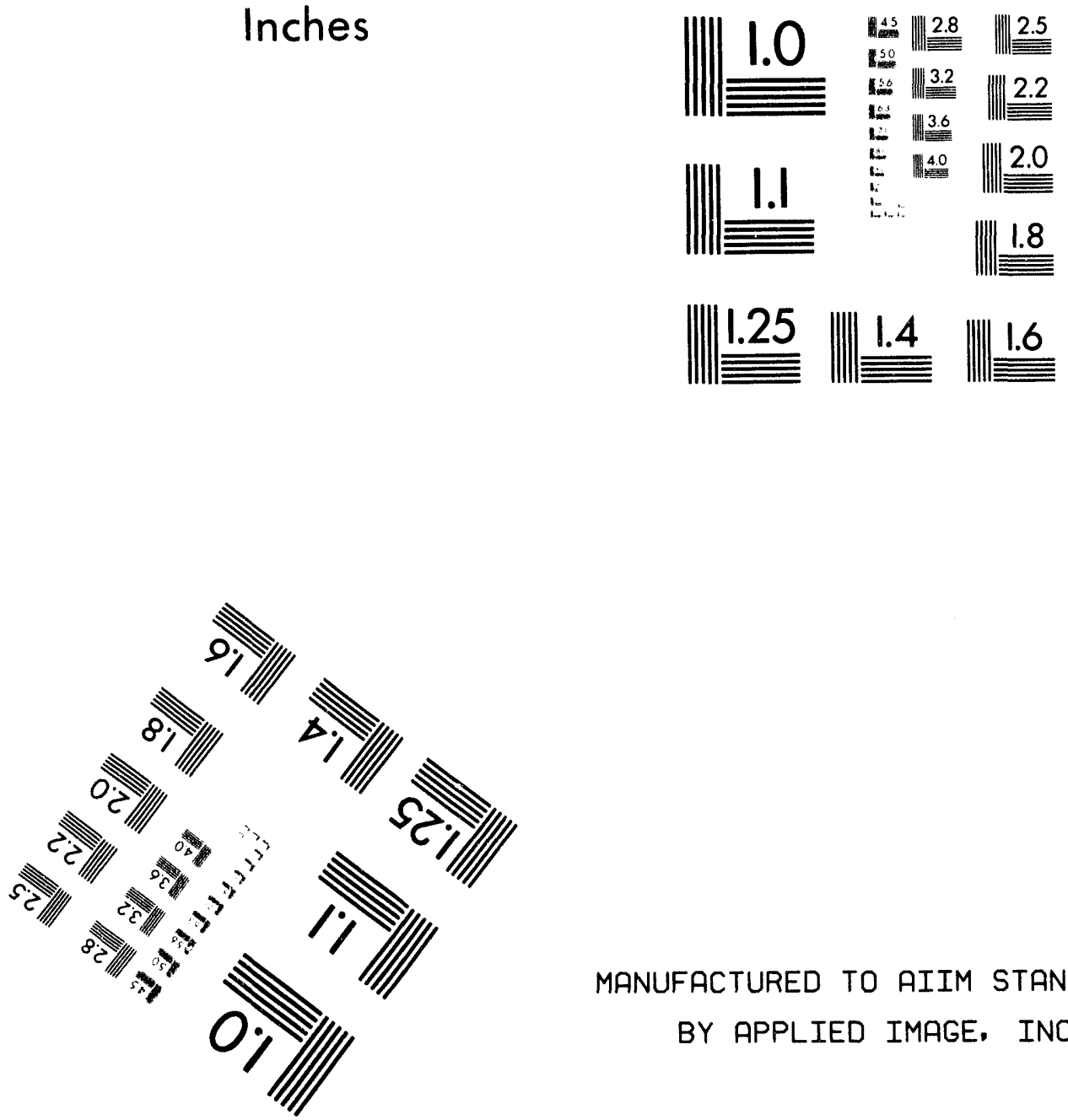

MANUFACTURED TO AIIM STANDARDS

BY APPLIED IMAGE, INC.

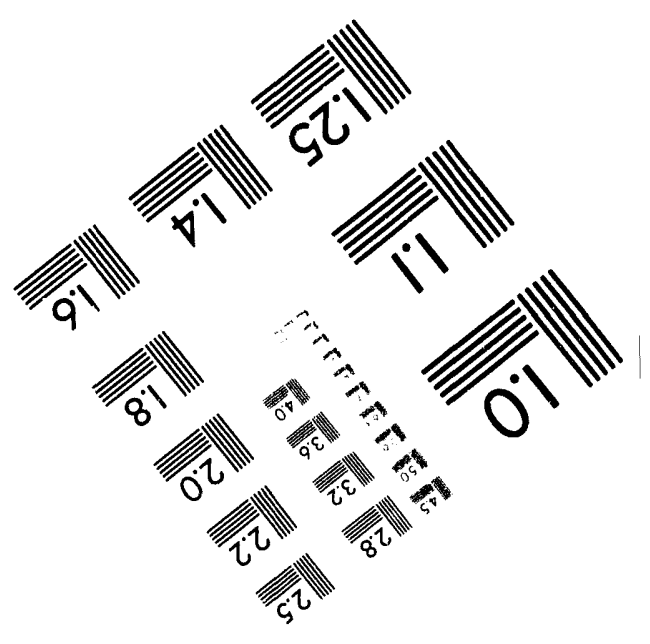



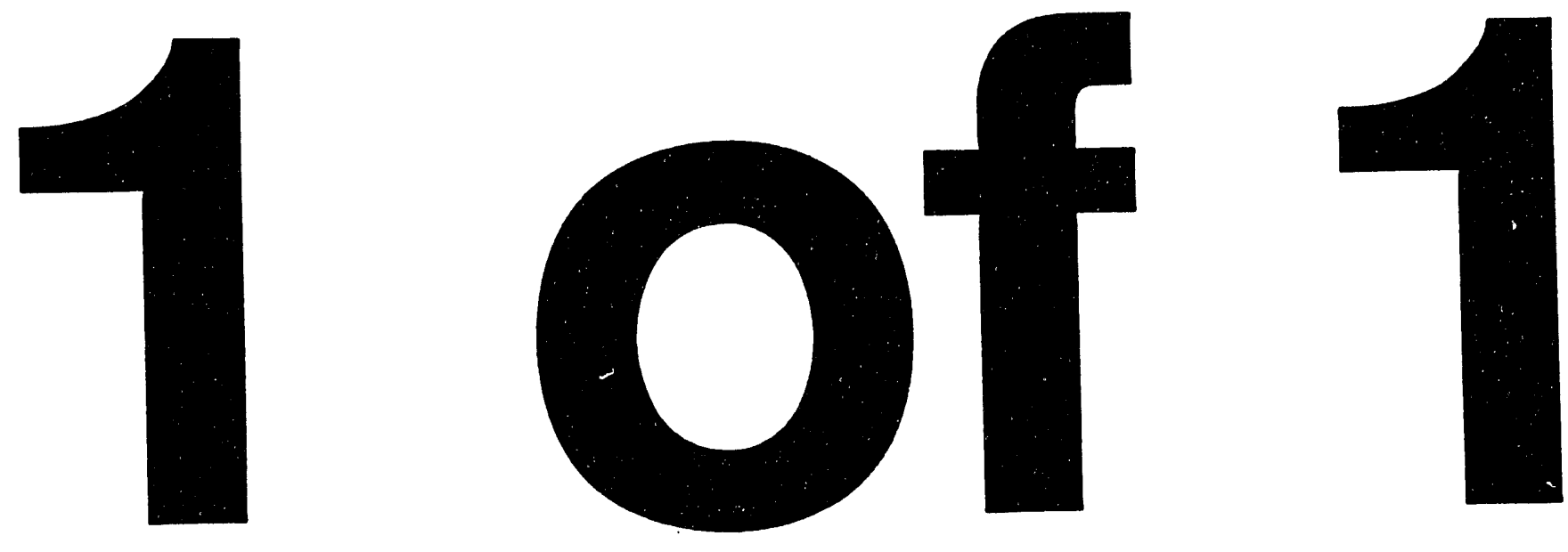


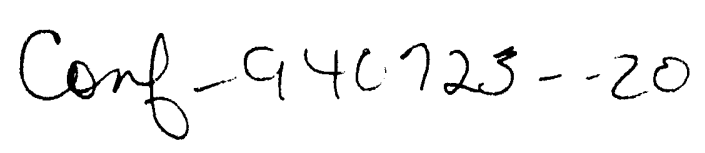

LA-UR- 94-2792
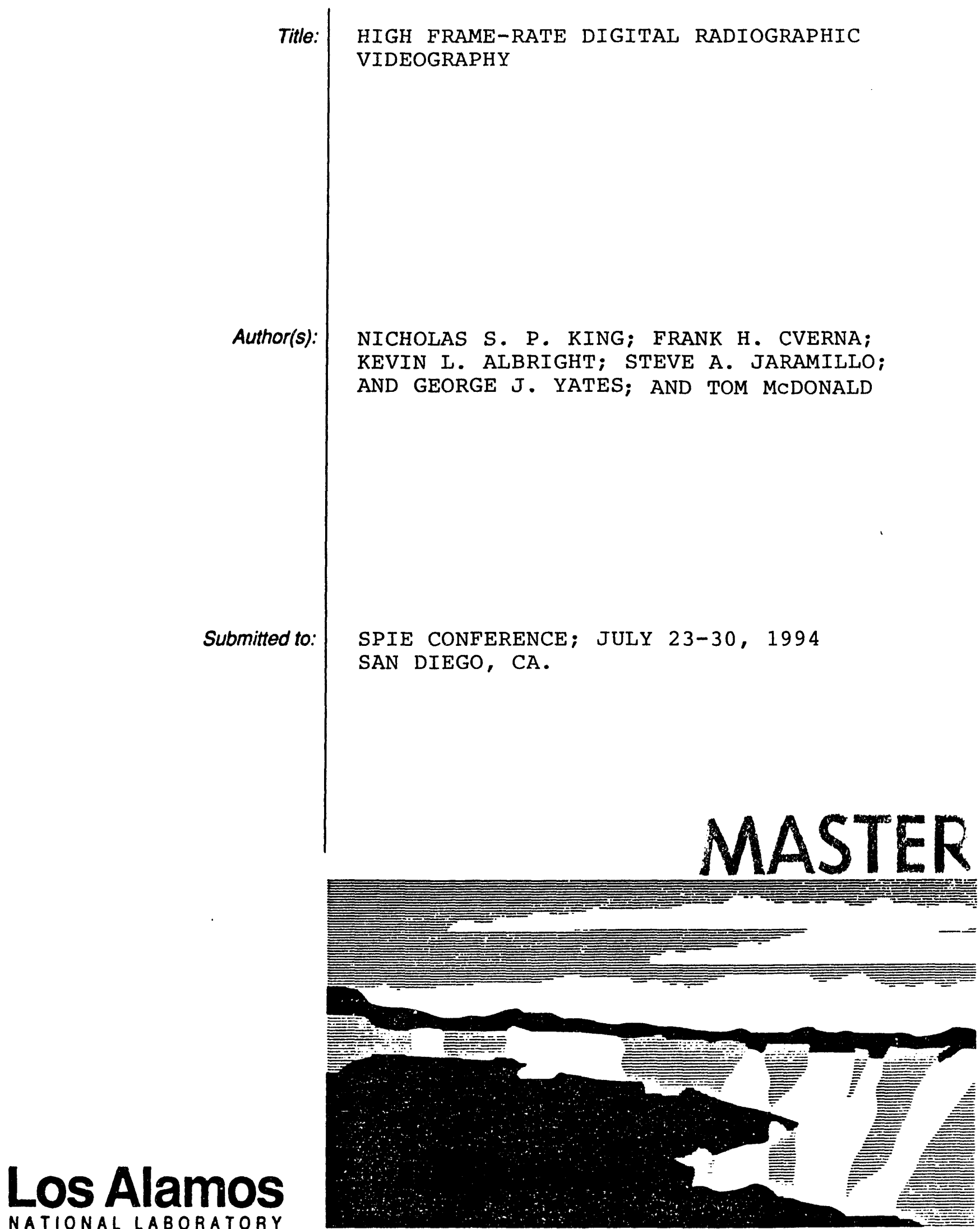

Los Alamos National Laboratory, an affirmative action/equal opportunity employer, is operated by the University of California for the U.S. Department of Energy under contract W-7405-ENG-26. By acceptance of this article, the publisher recognizes that the U.S. Government retains a nonexclusive, royalty-free license to publish or reproduce the published form of this contribution, or to allow others to do so, for U.S. Government purposes. The Los Alamos National Laboratory requests that the publisher identity this article as work pertormed under the auspices of the U.S. Departmerit of Energy. 
High Frame-Rate Digital Radiographic Videography

Nicholas S. P. King, Frank H. Cverna, Kevin I. Albright, Steve A. Jaramillo, George J. Yates and Thomas E. McDonald

LOs Alamor National Laboratory

Michael J. Flym and Scott Tashman

Henry Ford Health system

\begin{abstract}
High speed $x$-ray imaging can be an important tool for observing internal processes in a wide range of applications. In this paper we describe preliminary implementation of a system having the eventual goal of observing the internal dynamics of bone and joint reactions during loading. Two Los Alamos National Laboratory (LANL) gated and image intensified camera systems were used to record images from an $x$-ray image convertor tube to demonstrate the potential of high frame-rate digital radiographic videography in the analysis of bone and joint dynamics of the human body. Preliminary experiments were done at LANL to test the systems. Initial high frame-rate imaging (from 500 to 1000 frames/s) of a swinging pendulum mounted to the face of an X-ray image convertor tube demonstrated high contrast response and baseline sensitivity. The systems were then evaluated at the Motion Analysis Laboratory of Henry Ford Health Systems" Bone and Joint Center. Imaging of a 9 inch acrylic disk with embedded lead markers rotating at approximately 1000 RPM, demonstrated the system response to a high velocity/high contrast target. By gating the $\mathrm{P}-20$ phosphor image from the $\mathrm{X}$-ray image convertor with a second image intensifier (II) and using a 100 microsecond wide optical gate through the second II, enough prompt light decay from the $x$-ray image convertor phosphor had taken place to achieve reduction of most of the motion blurring. Measurement of the marker velocity was made by using video frames acquired at 500 frames/s. The data obtained from both experiments successfully demonstrated the feasibility of the technique. Several key areas for improvement are discussed along with salient test results and experiment details.
\end{abstract}

\title{
1. INTRODUCTION
}

$\mathrm{X}$-ray viewing of human anatomy is a common diagnostic tool for many diseases. Real time imaging of human subjects is commonly done using video recordings of the output phosphor of an image intensifier displayed at 30 full frames per second. In these application the $x$-ray beam is on continuously. Fast moving objects such as the human heart are typically imaged with pulsed $x$-ray sources to eliminate motion blur. Pulse widths of $4 \mathrm{msec}$ and pulse rates of 30 frames per second using cine film or digital camera recordings is commonly used in cardiac angiography. These cineangiographic recordings typicaly have high radiation dose per frame in order to produce images with low quantum noise. In general, good image quality and acceptable radiation dose to the patient can be obtained in a sequence of about 200 frames irrespective of the frame rate. 
Higher speed $x$-ray imaging techniques are required for some applications. For example, observation of knee joint compression starting with a heel strike and continuing for $100 \mathrm{~ms}$ is cerired for testing a hypothesis related to causality of osteoarthrosis (Ref. 1). The study of organ metabolic function might also benefit from high-speed imaging of radiolabled tracers that are used in nuclear medicine.

To implement high speed imaging, a new type of $x$-ray videography camera is required. The basic requirement of such a camera system is a frame rate of 1000 frames/s with an effective pixel array size of at least $256 \mathrm{X} 256$. The camera would typically be expected to record about 200 frames over a . 2 second period. An image gate width (shutter time) of no more that about 100 microseconds should be adequate to eliminate image smear.

Applying diagnostic technology developed for the underground nuclear testing program, we have carried out 500 frame/s imaging of a swinging pendulum to verify the basic concept. This was followed by demonstrated operation of the system at the Motion Analysis Laboratory, Henry Ford Health System, where a dynamic phantom was imaged.

\section{Proof of Concept Experiment}

As a proof of concept, we set up a high-speed imaging system at an $x$-ray testing site at Los Alamos using a $160 \mathrm{kV} x$-ray generator and a $16-i n c h \mathrm{x}-\mathrm{ray}$ image intensifier ( $x$-ray to light convertor). A high-speed image intensified camera was used to record the output image from the convertor. Three cameras were tested in this initial experiment. The first was a commercially available CCD camera operating at standard RS 170 television rates, which, although having too low a frame rate, provided a base line image for the tests. The second was the Los Alamos designed focus, projection, and scan, (FPS) 504 camera that was developed for the underground testing program in the 1970s and has proven to be a reliable diagnostic tool over the years (Ref 2). The third is a high-speed CCD camera, the GY6, which had evolved from a camera series that has been under development since the late $1980 \mathrm{~s}$ for use in the underground testing program and has been used with success in a number of experiments (Ref. 3).

The commercial camera did not have an intensifier and had a shutter speed of approximately $1 \mathrm{~ms}$. The FPS and GY cameras had image intensifiers with a gate (shutter time) of approximately $100 \mathrm{~ns}$. The GY camera was operated at approximately 650 frames/s with a pixel array size of $244 \mathrm{x} 380$. The FPS camera was configured to operate at 1000 frames/s with a resolution of 200 lines and a sweep speed of approximately 5 microseconds/line (an equivalent pixel array size of approximately $200 \mathrm{x} 200$ ). The $x$-ray source and $x$-ray convertor tube were operated effectively in the DC mode. A block diagram of the experiment is shown in Figure 1.

A satisfactory image was recorded by all three cameras. The image of the pendulum recorded by the commercial CCD camera was found to have a higher resolution than that of the GY camera because of its much lower frame rate. The image recorded by the FPS camera has about the same resolution as the commercial camera. Both the GY and 504 cameras were judged to be adequate for further testing at the Henry Ford Health Systems.

\section{Testing at the Bone and Joint Center Facility}

A high-speed imaging facility is being established at the Bone and Joint Center of the Henry Ford Health System to study the dynamics of joint loading, particularly the knee, and the forces on joints during normal activities such as walking or running. The experimental facility consists of dual $x$-ray sources, and dual $x$-ray image intensifiers. The $x$-ray source is single shot with a pulse length of $100 \mathrm{~ms}$, and is ojerated by a floor mounted force plate. Due to time constraints, a noise problem which developed in the GY camera could not be corrected so that only the 504 camera was tested in the 
facility. The 504 camera was configured to operate at 500 frames/s $(2$ $\mathrm{ms} /$ frame) and the optimal image intensifier gate width was 100 microseconds.

The block diagram of the experiment is basically the same as given in Figure 1. The target used for the dynamic tests was a 9-inch diameter rotating acrylic disk embedded with lead markers, attenuated by 0.75 inches of Aluminum. The speed of rotation was assumed to be 1000 revolutions per minute based on previous measurements. II sequence of 4 typical images taken by the 504 camera (out of a total of 40) of the rotating disk target is shown in Figure 2. The sequence is taken at $2 \mathrm{~ms}$ intervals during the $100 \mathrm{~ms} x$-ray pulse. A distinct image of the three 0.25 inch diameter lead markers, which are placed radially on the disk, is shown in the figure. The displacement of the row of markers during each $2 \mathrm{~ms}$ frame time is directly related to the velocity. Calculations were made for each marker type, which yielded the following velocities and speed of rotation:

$\begin{array}{llll}\text { Marker diameter } & \text { Mean Velocity } & \text { SD (m/s) } & \text { Speed (rpm) } \\ 0.08 \text { (in.) } & 8.75(\mathrm{~m} / \mathrm{s}) & 0.4 & 760 \\ 0.18 \text { (in.) } & 8.71(\mathrm{~m} / \mathrm{s}) & 0.49 & 756 \\ 0.25 \text { (in.) } & 8.48(\mathrm{~m} / \mathrm{s}) & 0.91 & 737\end{array}$

The disk has three sets of markers of different diameters. A line scan was taken through the innermost 0.25 inch diameter marker and the residual signal from the previous two frames. Note the two successively fainter images of the markers shown by the line scan. Note that the image polarity has been reversed from dark to light. The most distinct image of the marker is due to readout of the present target location. The fainter images are artifacts of the P-20 phosphor decay and target lag in the FPS photoconductive target. The noise seen to the right of each image is caused by the horizontal flyback of the FPS tube. The noise seen at the bottom is believed to be caused by the magnetic flux of the rotating anode of the $x$-ray tube.

\section{SUMMARY}

These experiments show that high-speed imaging of dynamic phenomena in the human anatomy is viable with the Los Alamos developed imaging systems. In experiments with human subjects, it would be desirable to replace the 100-ms single shot $x$-ray source with a pulsed source synchronized to emit only during the camera gating time. This would significantly reduce the $x$-ray dose. $\mathrm{X}$-ray pulse widths in the range of 10 to 100 microseconds are expected to be adequate for the application. A second technical issue that needs to be addressed is developing an image converter having a faster decay phosphor, such as P-46. This would provide a reduction in decay artifacts from the image captured by the high-speed camera. Finally, cameras capable of higher resolution such as 512×512, with a dynamic range of 1000:1, and nominally 1000 frames/s are needed for the accuracies required by the measurement.

The authors wish to acknowledge Kevin Dupre for his assistance in carrying out the experiments at Henry Ford Health System Bone and Joint Center, and Sumati Krishnan for calculations related to marker velocity.

\section{References}

1. Radin EL, Yang KH, Riegger C, et. al., "Relationship Between Lower Limb Dynamics and Knee Joint Pain", J. Orthop. Res., 9:398-405, 1991.

2. Yates GJ, Jaramillo SA, Holmes VH, Black JP, "Characterization of New FPS Vidicons for Scientific Imaging Applications", Los Alamos National Laboratory, LA-11035-MS, UC-37 June 1988.

3. Albright. KL, King NSP, Yates GJ, MCDonald TE, Turko BT, "An RS-170 to 700 frame per second CCD camera", PrOC. SPIE 2002. pp. 172-179. 


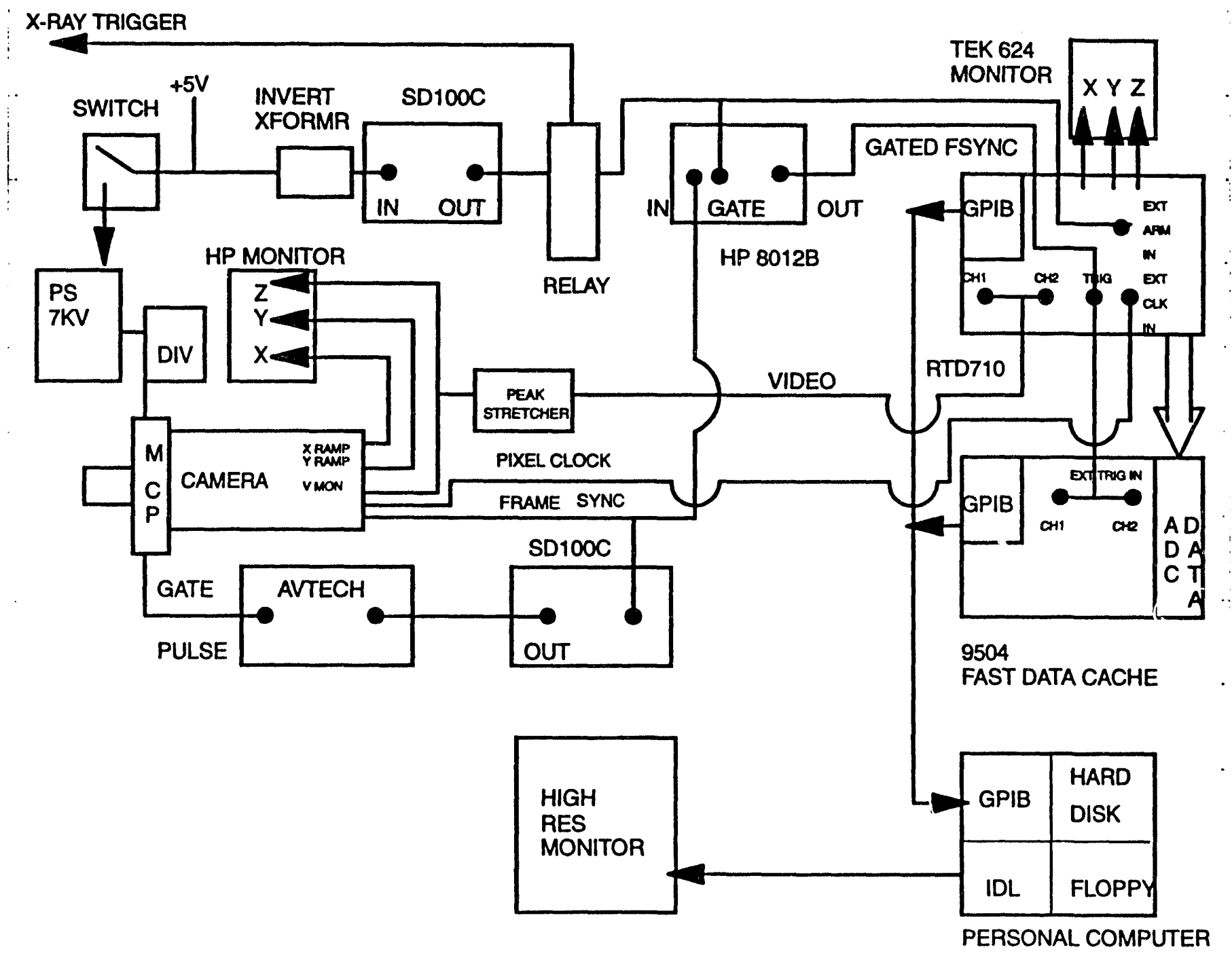

Figure 1 Block diagram of High-Speed $\mathrm{X}$-ray imaging experiment. Three cameras were initially tested, a commercially available CCD camera and the Los Alamos designed FPS and CCD cameras operating at 1000 frames/s and 650 frames/s respectively. 

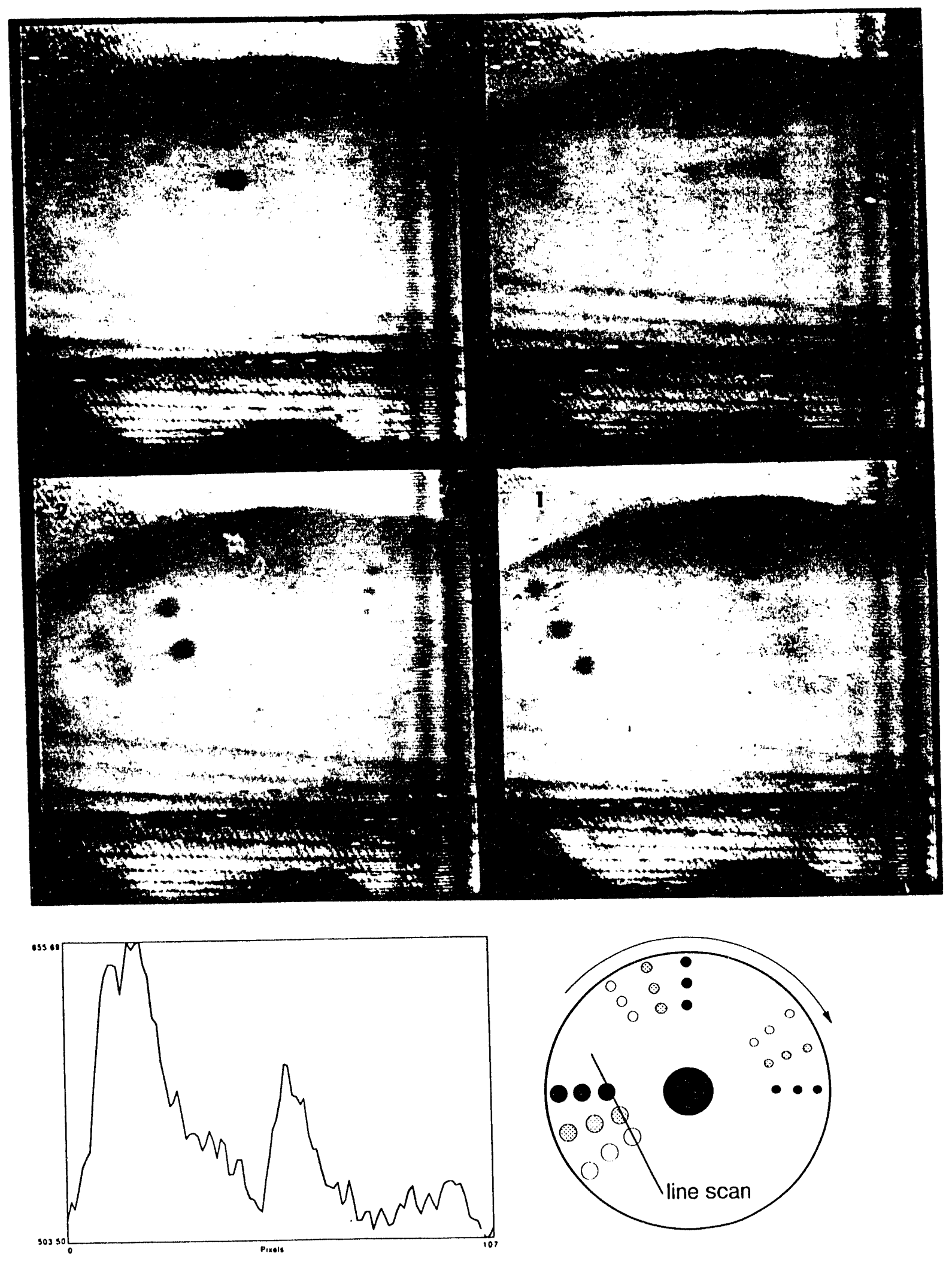

Figure 2 Typical sequence of data. Distinct images of three adjacent markers are shown radially on the disk. The most intense peak is the current image. Two falnter images across the 1 ine scan are due to the P-20 phosphor decay and phototarget decay after $2 \mathrm{~ms}$ and $4 \mathrm{~ms}$ respectively. 

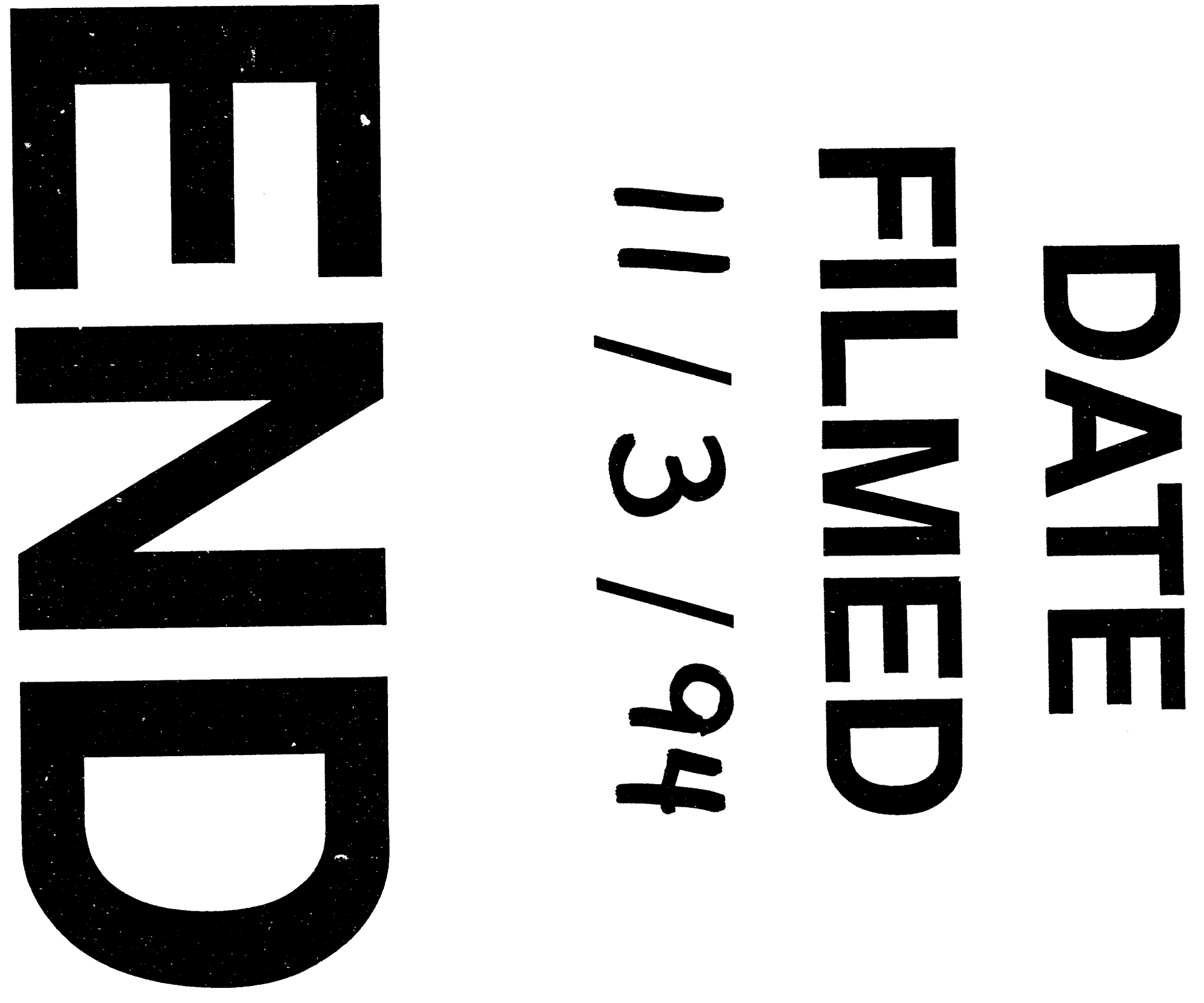
$\longrightarrow$ 